\title{
Values of Cytokines and Tryptophan Metabolites over a 12 Weeks Time Course in Patients with Depression and Somatoform Disorder
}

\author{
Daniela Krause $^{1, *}$, Verena B. Kirnich ${ }^{2, *}$, Theresa M. Stapf ${ }^{2}$, Anika Hennings ${ }^{3}$, Sabine Riemer ${ }^{3}$, Michael Riedel ${ }^{2}$, \\ Ralf Schmidmaier', Francisco Pedrosa Gil $^{5}$, Winfried Rief, ${ }^{3}$, Markus J. Schwarz ${ }^{2}$ \\ ${ }^{1}$ Department of Psychiatry and Psychotherapy, University Hospital, LMU Munich, ${ }^{2}$ Department of Laboratory Medicine, Ludwig Maximilians \\ University, Munich, ${ }^{3}$ Division of Clinical Psychology and Psychotherapy, Philipps University of Marburg, Marburg, ${ }^{4}$ Department of Hematology \\ and Oncology, Ludwig Maximilians University, Munich, ${ }^{5}$ Department of Psychiatry, Vogtland Hospital, Plauen, Germany
}

\begin{abstract}
Objective: Previous studies have suggested alterations in the kynurenine pathway as a major link between cytokine and neurotransmitter abnormalities in psychiatric disorders. Most of these studies used a cross-sectional case-control study design. However, knowledge is still lacking regarding the stability over time of kynurenine pathway metabolites and the functionally related cytokines. Therefore, we studied the stability of cytokines and tryptophan (TRP) parameters over a period of 12 weeks.

Methods: A total of 117 participants - 39 with major depression, 27 with somatoform disorder, and 51 healthy controls were enrolled. Four evaluations, including blood withdrawal and psychometric testing, were performed over a period of 12 weeks. We used ELISA to measure interleukin (IL)-6, IL-1 receptor antagonist (RA) and tumor necrosis factor $\alpha$ (TNF $\alpha$ ). High-performance liquid chromatography was used to analyze neurotransmitter variables, i.e. TRP, 5-hydroxyindoleacetic acid (5-HIAA), kynurenine (KYN), 3-OH-kynurenine (3-HK), and kynurenic acid (KYNA).

Results: We found no significant fluctuations of TRP, its metabolites (5-HIAA, KYN, KYNA, and 3-HK), or the cytokines (IL-1RA, IL-6, and TNF $\alpha$ ) in any of the groups over the 12 weeks.

Conclusion: To our knowledge, this is the first longitudinal study performed in psychiatric patients to verify the stability and consequently the reliability of the biological parameters we investigated. Our data indicate that TRP metabolites and cytokines are reliable biological parameters in psychiatric research because they do not fluctuate significantly over time.
\end{abstract}

KEY WORDS: Cytokines; Tryptophan; Depression; Somatoform disorders; Kynurenine; Kynurenic acid.

\section{INTRODUCTION}

In the field of psychoneuroimmunological research, evidence has been accumulating for reciprocal communication pathways between the nervous, endocrine, and immune systems. ${ }^{1)}$ As a result, there is growing interest in whether and in which way these interactions are involved in the development of psychiatric disorders. Proinflammatory cytokines, as described recurrently in major de-

Received: April 24, 2017 / Revised: August 31, 2017

Accepted: October 24, 2017

Address for correspondence: Daniela Krause, MD

Department of Psychiatry, Ludwig Maximilians University,

Nußbaumstr. 7, 80336 Munich, Germany

Tel: +49-89-4400-55511, Fax: +49-89-4400-73240

E-mail: Daniela.Krause@med.uni-muenchen.de

ORCID: https://orcid.org/0000-0003-0966-2521

*These authors contributed equally to this publication. pression $(\mathrm{MD}){ }^{2-4)}$ are supposed to be related to an enhanced degradation of plasma tryptophan (TRP) via the kynurenine pathway. ${ }^{5,6)}$ There is robust evidence that interactions of the immune system and serotonergic system, via indolamine 2,3-dioxygenase, the rate-limiting enzyme in the kynurenine pathway, play a key role in MD. ${ }^{7)}$ Not only depression, also Alzheimer's disease ${ }^{8)}$ and especially somatoform disorder (SFD) has been shown to be associated with specific biomarkers of inflammation and cell-mediated immune activation, that are correlated with changes in the TRP catabolite pathway. ${ }^{9)}$ Taken these findings together, it is further strengthened that depression and SFD are two frequently overlapping clinical conditions having both been related to immunological changes. $^{10)}$

Recently, psychobiological studies in depression and

(c) This is an Open-Access article distributed under the terms of the Creative Commons Attribution Non-Commercial License (http://creativecommons.org/licenses/by-nc/4.0) which permits unrestricted non-commercial use, distribution, and reproduction in any medium, provided the original work is properly cited. 
SFD have had a huge impact on research. Especially depression research has lately very much focused on the pro- and anti-inflammatory trias of interleukin (IL)-6, tumor necrosis factor (TNF) $\alpha$ and IL-1 receptor antagonist (RA), as well as on the investigation of kynurenine pathway alterations with predominance of the neurotoxic pathway. ${ }^{11)}$ However, the vast majorities of studies followed a cross-sectional case-control design and thus were unable to prove the stability of the investigated parameters over time. Therefore, the main aim of our study was to measure the most relevant biological features of depression and somatization in a longitudinal study. To our knowledge data about the longitudinal course and stability of pro-inflammatory cytokines and TRP metabolites is rare in patients suffering from MD and SFD and compared to healthy controls $(\mathrm{HC})$. One recent study investigated the cytokines IL-6 and IL-18 already twice (baseline two weeks post stroke and then again one year later) in post-stroke depression. It was concluded that higher IL- 6 and IL-1 8 values were associated with depressive disorders within two weeks and at one year after stroke. ${ }^{12)}$ Furthermore there is another study that measured IL-6, TNF $\alpha$ and high sensitivity C-reactive protein (hsCRP) twice over the course of five years and calculated the relationship to depressive symptoms in men. For the two cytokines none was significantly associated with depression, only for hsCRP an association to depressive symptoms could be identified. ${ }^{13)}$ However, knowledge about the long-term stability of these biological parameters is essential to validate the findings of previous and future cross-sectional studies Therefore, the main aim of the present study was to investigate the stability of the pro-inflammatory cytokines IL- 6 and TNF $\alpha$ and the anti-inflammatory IL-1 RA in MD, SFD, and HC over a period of 12 weeks. Because of the known interactions between cytokines and neurotransmitter systems, we assessed the stability of both cytokines and TRP metabolites, thereby focusing on the kynurenine pathway.

\section{METHODS}

\section{Sample}

A total of 117 subjects, comprising 39 outpatients with depression, 27 outpatients with SFD (defined according to Somatoform Symptom Index [SSI] 6 out of 8), and 51 age-matched $\mathrm{HC}$, were recruited by public advertise- ments, press releases in local newspapers, and leaflets in clinics, pharmacies, and universities. This study was part of an experimental approach to investigate the effect of physical exercise on symptom severity in $\mathrm{MD}$ and SFD. ${ }^{14,15)}$ The study protocol was approved by the ethics committees of the Ludwig Maximilian University, Munich, and the German Psychological Society. All participants provided written informed consent. As an incentive, all participants received a small financial compensation for completing the study. All participants were pre-screened in a short telephone interview. The effect size of the study was calculated according to Cohen's $d$. For medium effect sizes at a $3 \times 2 \times 2 \times 2 \times 6$ factor design (group $\times$ sex $\times$ intervention $\times$ order $\times$ time point) and an error probability of $p<$ 0.05 a total of $n=108$ study participants is needed. During the 12 week course of the study the following drop-out rates were observed: 3 people in MD group, 2 people in the SFD group and 4 people in $\mathrm{HC}$.

\section{Psychological Measures}

Suitable participants took part in a structured clinical interview (SCID; German version) ${ }^{16)}$ for Diagnostic and Statistical Manual of Mental Disorders of the American Psychiatric Association, fourth edition (DSM-IV) to confirm present and lifetime diagnoses as well as co-morbid Axis I disorders. Interviewers at both of the study centers were physicians or psychologists familiar with conducting SCID and experienced in making psychiatric diagnoses. Sociodemographic variables, like body mass index (BMI) and physical activity (assessed with the "Freiburger Fragebogen zur körperlichen Aktivität," FFKA [Freiburger Questionnaire on physical activity]), were recorded because of their possible influence on biological parameters. Further questionnaires about personality (SCID II) and social support (F-Sozu [German Questionnaire on Social Support]) were completed. Exclusion criteria were organic illnesses affecting the central nervous system or immune system, pregnancy, psychotic symptoms, alcohol or drug abuse, and current psychotherapy. During the study, we excluded also patients who developed a medical condition, e.g. a common cold. To reduce the number of missing values, we shifted the measurement for 4 weeks, so that women were again in the same period of their cycle. For the patient groups, co-morbidity of MD and SFD was an additional exclusion criterion. Furthermore, a weak somatization syndrome with four (men) or six 
(women) symptoms (SSI-4/6) was an exclusion criterion in the depression group. Oral contraceptives were permitted, but we excluded persons taking other immune-modulating medication (e.g., corticosteroids). A past or present psychiatric diagnosis according to DSM-IV was an exclusion criterion in the $\mathrm{HC}$ group.

SFD is characterized by bodily symptoms that cannot be explained by a clear organic pathology or known physiological mechanisms. Typically somatoform symptoms are long-lasting and disabling. ${ }^{17,18)}$ We used the SSI-6/8 to define SFD, because of previous claims that the classification of SFDs according to DSM-IV is too strict and does not give a good representation of the relevance to healthcare of patients with multiple somatoform symptoms. ${ }^{19)}$ By using an empirical approach to study the definition of SFD, Rief and Hiller ${ }^{20)}$ found that a cut-off score of seven to eight symptoms is valid for differentiating between patients with high and low levels of disability. The SSI-6/8 requires that patients have at least eight (men only six) of the 33 complaints relevant for the classification of SFD according to DSM-IV.

MD was diagnosed strictly according to DSM-IV criteria, i.e. the presence of a period of at least 2 weeks of depressed mood or anhedonia or both in combination with at least five symptoms like somatic disturbances (change in sleep, appetite, energy level, and psychomotor functioning) and cognitive disturbances (feeling of inappropriate guilt, worthlessness, trouble concentrating and making decisions, and suicidal thoughts) that cause clinical significant distress or impairment in everyday functioning. ${ }^{16)}$

\section{Procedures}

After completion of the diagnostic session, we arranged the first blood withdrawal $(t 1)$. Blood samples were taken between 8 AM and 9 AM exactly every four, eight, and twelve weeks thereafter $(t 2-t 4)$. Patients were instructed to avoid alcohol and exercise for 24 hours before blood withdrawal. After blood sampling participants completed the following self-rating scales: Beck Depression Inventory (BDI) to assess dpression severity, ${ }^{21)}$ Screening for Somatoform Symptoms-7 (SOMS-7) to examine somatoform symptom severity, and Symptom Checklist-90Revised (SCL-90-R) to detect global psychopathological impairment. ${ }^{22)}$ Nutrition and sleep were assessed with protocols to control for potential sleep disturbances and nutrition abnormalities that could affect cytokine and neurotransmitter levels. At $t 1$ and $t 4$, the level of chronic stress was measured with the Trier Inventory for Chronic Stress in its Shortform (TICS-K), and at $t 4$, somatoform symptoms during the last two years were assessed with the SOMS-2. ${ }^{23)}$ Because of the reported influence of physical activity on the immune system, we assessed the participants' typical amount of physical activity at the onset of the study with the FFKA. ${ }^{24)}$

\section{Analysis of blood samples}

Blood samples (two $7.5 \mathrm{ml}$ serum tubes) were collected between $8 \mathrm{AM}$ and $9 \mathrm{AM}$. Immediately after collection, samples were allowed to clot for 30 minutes and then centrifuged; serum was stored at $-80^{\circ} \mathrm{C}$ until being thawed for assay. All blood samples were assayed in a single run at the end of the data collection.

Analysis of TRP metabolites with high performance liquid chromatography

High performance liquid chromatography (HPLC; Waters Co., Milford, MA, USA) was used to measure serum TRP, 5-hydroxyindoleacetic acid (5-HIAA), kynurenine (KYN), 3-OH-kynurenine (3-HK), and kynurenic acid (KYNA). All TRP metabolites were measured by a previously described HPLC method. ${ }^{25)}$ To avoid operator bias, tubes were coded only with numbers so that the laboratory technician was blinded to group.

\section{Analysis of cytokines with quantitative enzyme-linked immunosorbent assay}

The serum samples were analyzed for the cytokines IL-1 RA, IL-6, and TNF $\alpha$. All assays were carried out with quantitative enzyme-linked immunosorbent assay (ELISA) kits in accordance with the manufacturers' instructions (Human IL-6 Quantikine HS, Human TNF $\alpha$ Quantikine HS; Human IL-1 RA Quantikine, all from R\&D Systems, Minneapolis, MN, USA). A highly sensitive ELISA was used for IL- 6 and TNF $\alpha$. According to the manufacturer, the intra-assay coefficient of variation was between $6.9 \%$ and $7.8 \%$ for IL-6, between $3.1 \%$ and $8.5 \%$ for TNF $\alpha$, and between $3.7 \%$ and $7.3 \%$ for IL-1 RA, with a minimum detectable amount of $0.039 \mathrm{pg} / \mathrm{ml}$ for IL-6, $0.106 \mathrm{pg} / \mathrm{ml}$ for TNF $\alpha$, and $6.26 \mathrm{pg} / \mathrm{ml}$ for IL-1 RA. 


\section{Statistical Analysis}

SPSS Statistics software (version 23; IBM Corp., Armonk, NY, USA) was used for statistical procedures. The chi-square test was used to check for potential demographic group differences in categorical variables; ANOVA or Kruskal-Wallis test, for potential demographic group differences in ordinal and interval variables. Further, all data were tested for normal distribution by plotting the values and with the Kolmogorov-Smirnov test. Since most psychometric variables and immunological parameters were distributed non-parametrically, we used mainly non-parametric tests. We applied the Friedman rank sum test and Spearman's correlations to analyze the stability of cytokines and TRP metabolites during the 12 weeks. A Pearson product-moment correlation coefficient was computed to assess the relationship between the kynurenine system and cytokines. Interactions between biological parameters and socio- demographic characteristics and between biological parameters and psychometric results were analyzed with Spearman rank coefficients. A $p<0.05$ was considered as statistically significant. Because of the explorative character of our study, we did not use a Bonferroni adjustment. ${ }^{26)}$

\section{RESULTS}

\section{Sample Characteristics}

Thirty-nine patients with MD (age, 32.6 \pm 12.6 years), 27 patients with SFD (age, 38.8 \pm 14.29 years), and $51 \mathrm{HC}$ (age, $36.51 \pm 13.18$ years) were included in the study. In all groups, more than $60 \%$ of the participants were female and over $72 \%$ had at least high school education. The groups did not differ in terms of age, sex distribution, smoking, BMI, physical activity, or nutrition (kcal and proteins). In total, $23.1 \%$ of patients with MD were taking antidepressants (mainly SSRIs). The SFD group was treat-

Table 1. Characteristics of patients with somatoform disorder (SFD) or major depression (MD) and healthy controls (HC)

\begin{tabular}{|c|c|c|c|c|}
\hline Characteristic & SFD group $(n=27)$ & MD group $(n=39)$ & HC group $(n=51)$ & Significance level \\
\hline \multicolumn{5}{|l|}{ Sociodemographic data } \\
\hline Age (yr) & $38.8 \pm 14.29$ & $32.64 \pm 12.58$ & $36.51 \pm 13.18$ & $F=1.88 ; \mathrm{NS}$ \\
\hline Women & $20(74.1)$ & $24(61.5)$ & $33(64.7)$ & $\mathrm{Chi}^{2}=1.16 ; \mathrm{NS}$ \\
\hline High school education & $18(66.7)$ & $30(76.9)$ & $43(84.3)$ & $\mathrm{Chi}^{2}=5.59 ; \mathrm{NS}$ \\
\hline Occupation & $24(88.9)$ & $36(92.3)$ & $43(82.4)$ & $\mathrm{Chi}^{2}=0.50 ; \mathrm{NS}$ \\
\hline Smoking status & $4(14.8)$ & $15(38.5)$ & $16(31.4)$ & $\mathrm{Chi}^{2}=4.62 ; \mathrm{NS}$ \\
\hline Adequate physical activity & $11(40.7)$ & $12(30.8)$ & $26(51.0)$ & $\mathrm{Chi}^{2}=8.67 ; \mathrm{NS}$ \\
\hline Antidepressants & $4(14.8)$ & $9(23.1)$ & $0(0)$ & $\mathrm{Chi}^{2}=0.13 ; \mathrm{NS}^{\mathrm{a}}$ \\
\hline Body mass index $\left(\mathrm{kg} / \mathrm{m}^{2}\right)$ & $24.11 \pm 4.80$ & $24.72 \pm 4.83$ & $24.05 \pm 5.47$ & $\mathrm{Chi}^{2}=0.97 ; \mathrm{NS}$ \\
\hline \multicolumn{5}{|l|}{ Psychiatric comorbidity } \\
\hline Generalized anxiety disorder & $2(7.4)$ & $2(5.1)$ & - & $\mathrm{Chi}^{2}=0.12 ; \mathrm{NS}^{\mathrm{a}}$ \\
\hline Obsessive-compulsive disorder & $3(11.1)$ & $1(2.6)$ & - & $\mathrm{Chi}^{2}=1.94 ; \mathrm{NS}^{\mathrm{a}}$ \\
\hline Panic disorder & $4(14.8)$ & $6(15.4)$ & - & $\mathrm{Chi}^{2}=0.01 ; \mathrm{NS}^{\mathrm{a}}$ \\
\hline Posttraumatic stress disorder & $0(0)$ & $5(12.8)$ & - & $\mathrm{Chi}^{2}=3.79 ; \mathrm{NS}^{\mathrm{a}}$ \\
\hline Social phobia & $4(14.8)$ & $7(17.9)$ & - & $\mathrm{Chi}^{2}=0.14 ; \mathrm{NS}^{\mathrm{a}}$ \\
\hline Specific phobia & $2(7.4)$ & $2(5.1)$ & - & $\mathrm{Chi}^{2}=0.12 ; \mathrm{NS}^{\mathrm{a}}$ \\
\hline \multicolumn{5}{|l|}{ Psychopathological measures } \\
\hline F-Sozu & $59.5 \pm 9.3$ & $56.8 \pm 12.95$ & $65.2 \pm 8.0$ & $\mathrm{Chi}^{2}=13.8 ; p=0.001^{* * *}$ \\
\hline & & & & MD vs. SSI: $\mathrm{T}=0.69 ; \mathrm{NS}$ \\
\hline Depression, BDI & $13.78 \pm 8.57$ & $21.63 \pm 9.52$ & $3.06 \pm 4.25$ & $\mathrm{Chi}^{2}=69.16 ; p=0.000^{* * *}$ \\
\hline Psychological distress, SCL 90-R, GSI & $0.78 \pm 0.52$ & $1.01 \pm 0.50$ & $0.13 \pm 0.17$ & $\mathrm{Chi}^{2}=60.18 ; p=0.000^{* * *}$ \\
\hline \multicolumn{5}{|l|}{ Somatization severity indices } \\
\hline Total SOMS-7 & $29.31 \pm 17.45$ & $8.00 \pm 9.52$ & $5.86 \pm 5.63$ & $\mathrm{Chi}^{2}=47.90 ; p=0.000^{* * *}$ \\
\hline Total SOMS-2 & $26.00 \pm 9.93$ & $23.88 \pm 9.52$ & $5.74 \pm 7.68$ & $\mathrm{Chi}^{2}=107.33 ; p=0.001^{* * *}$ \\
\hline \multicolumn{5}{|l|}{ Stress Evaluation } \\
\hline TICS-K & $21.73 \pm 9.62$ & $26.43 \pm 7.76$ & $9.53 \pm 6.48$ & $\mathrm{Chi}^{2}=103.48 ; p=0.009^{* *}$ \\
\hline
\end{tabular}

Values are presented as mean \pm standard deviation or number (\%).

Group differences were calculated with analyses of variance and chi-square tests as appropriate.

NS, not significant; SSI, Somatoform Symptom Index; BDI, Beck Depression Inventory; SCL-90 R, Symptom Check List-90 Revised; GSI, Global Severity Index; SOMS, Screening for Somatoform Symptoms; TICS-K, Trier Inventory for Chronic Stress in its Shortform.

The right column includes significant results $(p<0.05) ;{ }^{*} p<0.05, * * p<0.01,{ }^{* * *} p<0.001$; ${ }^{a}$ comparison between patient groups. 
ed only in $14.8 \%$ with medication (antidepressants, lithium and anticonvulsant). The most frequent comorbidities were social phobia and panic disorder.

Detailed characteristics of the study sample are presented in Table 1.

\section{Longitudinal Examination of Cytokines and TRP Metabolites}

The Friedman rank sum test demonstrated stability of all investigated biological parameters over the course of 12 weeks: significant fluctuations were not observed in either of the patient groups or in the $\mathrm{HC}$ group. Detailed results for TRP and its metabolites are presented in Table 2. The immune markers IL-6, TNF $\alpha$, and IL-1 RA were also stable over the 12 weeks (Table 3). To check for un- systematic variations, we also performed Spearman's correlations between the single measurements. These tests (Table 4) also showed no fluctuations of the investigated parameters. Further, we performed a subgroup analysis of the parameters to verify their stability (in females and in participants without psychiatric medication), which were in line with our results (data not shown). Box plot analysis also showed high stability. Only the $\mathrm{HC}$ group contained sporadic outliers in the cytokines IL-1 RA and IL- 6 as well as in the serotonin degradation product 5-HIAA. Detailed results are shown in Tables 2 to 4 .

\section{Psychopathology}

As expected, somatization scores were higher in SFD patients and depression scores higher in MD patients.

Table 2. Longitudinal analysis of tryptophan and kynurenine metabolites

\begin{tabular}{|c|c|c|c|c|c|c|}
\hline Biological parameter & Group & $t 1$ & $t 2$ & $t 3$ & $t 4$ & Friedman rank sum test \\
\hline \multirow[t]{4}{*}{ Tryptophan (ng/mg) } & $M D(n=37)$ & $16.80 \pm 3.98$ & $16.82 \pm 4.60$ & $16.93 \pm 4.13$ & $16.17 \pm 3.83$ & $\mathrm{Chi}^{2}=1.24 ; \mathrm{df}=3 ; p=0.74$ \\
\hline & $\operatorname{SFD}(n=26)$ & $15.78 \pm 3.00$ & $15.67 \pm 3.38$ & $15.33 \pm 3.04$ & $15.09 \pm 2.85$ & $\mathrm{Chi}^{2}=1.99 ; \mathrm{df}=3 ; p=0.58$ \\
\hline & $\mathrm{HC}(\mathrm{n}=43)$ & $16.85 \pm 4.59$ & $16.92 \pm 4.41$ & $16.84 \pm 4.65$ & $16.17 \pm 3.55$ & $\mathrm{Chi}^{2}=1.80 ; \mathrm{df}=3 ; p=0.62$ \\
\hline & Total $(n=106)$ & $16.57 \pm 4.03$ & $16.58 \pm 4.24$ & $16.50 \pm 4.14$ & $15.91 \pm 3.50$ & $\mathrm{Chi}^{2}=3.52 ; \mathrm{df}=3 ; p=0.32$ \\
\hline \multirow[t]{4}{*}{ 5-HIAA (ng/mg) } & $M D(n=37)$ & $2.66 \pm 3.03$ & $2.75 \pm 3.34$ & $2.67 \pm 3.11$ & $2.45 \pm 3.17$ & $\mathrm{Chi}^{2}=1.45 ; \mathrm{df}=3 ; p=0.69$ \\
\hline & $\operatorname{SFD}(n=25)$ & $4.68 \pm 6.35$ & $6.10 \pm 10.65$ & $4.21 \pm 5.25$ & $3.81 \pm 4.59$ & $\mathrm{Chi}^{2}=5.68 ; \mathrm{df}=3 ; p=0.13$ \\
\hline & $\mathrm{HC}(\mathrm{n}=43)$ & $3.97 \pm 5.30$ & $5.35 \pm 13.08$ & $4.96 \pm 13.31$ & $3.52 \pm 5.76$ & $\mathrm{Chi}^{2}=1.02 ; \mathrm{df}=3 ; p=0.80$ \\
\hline & Total $(n=105)$ & $3.68 \pm 4.95$ & $4.61 \pm 10.06$ & $3.97 \pm 9.07$ & $3.22 \pm 4.70$ & $\mathrm{Chi}^{2}=4.21 ; \mathrm{df}=3 ; p=0.24$ \\
\hline \multirow[t]{4}{*}{ Kynurenine (ng/mg) } & $\mathrm{MD}(\mathrm{n}=37)$ & $433.17 \pm 93.36$ & $440.55 \pm 102.08$ & $442.24 \pm 91.84$ & $436.23 \pm 111.49$ & $\mathrm{Chi}^{2}=0.80 ; \mathrm{df}=3 ; p=0.85$ \\
\hline & $\operatorname{SFD}(n=26)$ & $458.69 \pm 151.51$ & $435.04 \pm 110.75$ & $448.32 \pm 140.24$ & $435.78 \pm 124.36$ & $\mathrm{Chi}^{2}=1.25 ; \mathrm{df}=3 ; p=0.74$ \\
\hline & $\mathrm{HC}(\mathrm{n}=43)$ & $464.70 \pm 107.99$ & $455.28 \pm 91.93$ & $456.89 \pm 96.25$ & $459.58 \pm 103.12$ & $\mathrm{Chi}^{2}=0.29 ; \mathrm{df}=3 ; p=0.96$ \\
\hline & Total $(n=106)$ & $452.22 \pm 115.42$ & $445.18 \pm 99.74$ & $449.67 \pm 106.40$ & $445.59 \pm 111.05$ & $\mathrm{Chi}^{2}=1.19 ; \mathrm{df}=3 ; p=0.76$ \\
\hline \multirow{4}{*}{$\begin{array}{l}\text { 3-OH-kynurenine } \\
\text { (ng/mg) }\end{array}$} & $\mathrm{MD}(\mathrm{n}=37)$ & $8.64 \pm 3.20$ & $8.47 \pm 3.0$ & $9.20 \pm 3.4$ & $8.75 \pm 4.26$ & $\mathrm{Chi}^{2}=3.38 ; \mathrm{df}=3 ; p=0.34$ \\
\hline & SFD $(n=25)$ & $10.47 \pm 5.10$ & $10.17 \pm 4.02$ & $9.98 \pm 4.17$ & $10.54 \pm 5.40$ & $\mathrm{Chi}^{2}=0.16 ; \mathrm{df}=3 ; p=0.98$ \\
\hline & $\mathrm{HC}(\mathrm{n}=43)$ & $9.65 \pm 3.84$ & $9.73 \pm 3.70$ & $9.82 \pm 4.16$ & $9.69 \pm 4.03$ & $\mathrm{Chi}^{2}=0.50 ; \mathrm{df}=3 ; p=0.92$ \\
\hline & Total $(n=105)$ & $9.35 \pm 3.88$ & $9.40 \pm 3.57$ & $9.59 \pm 3.83$ & $9.54 \pm 4.50$ & $\mathrm{Chi}^{2}=2.07 ; \mathrm{df}=3 ; p=0.56$ \\
\hline \multirow[t]{4}{*}{ Kynurenic acid (ng/mg) } & $\mathrm{MD}(\mathrm{n}=37)$ & $11.06 \pm 10.15$ & $11.51 \pm 10.02$ & $11.72 \pm 6.83$ & $12.68 \pm 13.60$ & $\mathrm{Chi}^{2}=3.67 ; \mathrm{df}=3 ; p=0.30$ \\
\hline & $\operatorname{SFD}(n=25)$ & $11.02 \pm 4.87$ & $9.86 \pm 5.49$ & $10.26 \pm 5.19$ & $10.39 \pm 4.93$ & $\mathrm{Chi}^{2}=2.13 ; \mathrm{df}=3 ; p=0.55$ \\
\hline & $\mathrm{HC}(\mathrm{n}=43)$ & $10.41 \pm 6.65$ & $10.42 \pm 5.30$ & $10.30 \pm 5.79$ & $11.18 \pm 11.23$ & $\mathrm{Chi}^{2}=0.98 ; \mathrm{df}=3 ; p=0.81$ \\
\hline & Total $(n=105)$ & $10.78 \pm 7.69$ & $10.67 \pm 7.31$ & $10.79 \pm 6.03$ & $11.52 \pm 11.02$ & $\mathrm{Chi}^{2}=0.18 ; \mathrm{df}=3 ; p=0.98$ \\
\hline \multirow{4}{*}{$\begin{array}{l}\text { Kynurenic acid/ } \\
\text { kynurenine }\end{array}$} & $M D(n=37)$ & $0.026 \pm 0.028$ & $0.026 \pm 0.022$ & $0.026 \pm 0.015$ & $0.031 \pm 0.041$ & $\mathrm{Chi}^{2}=3.29 ; \mathrm{df}=3 ; p=0.35$ \\
\hline & $\operatorname{SFD}(n=25)$ & $0.025 \pm 0.011$ & $0.023 \pm 0.012$ & $0.024 \pm 0.010$ & $0.024 \pm 0.011$ & $\mathrm{Chi}^{2}=2.71 ; \mathrm{df}=3 ; p=0.44$ \\
\hline & $\mathrm{HC}(\mathrm{n}=43)$ & $0.022 \pm 0.013$ & $0.023 \pm 0.010$ & $0.023 \pm 0.012$ & $0.024 \pm 0.017$ & $\mathrm{Chi}^{2}=1.72 ; \mathrm{df}=; 3 \quad p=0.63$ \\
\hline & Total $(n=105)$ & $0.024 \pm 0.019$ & $0.024 \pm 0.016$ & $0.024 \pm 0.013$ & $0.027 \pm 0.027$ & $\mathrm{Chi}^{2}=0.19 ; \mathrm{df}=3 ; p=0.98$ \\
\hline \multirow{4}{*}{$\begin{array}{l}\text { 3-OH-kynurenine/ } \\
\text { kynurenine }\end{array}$} & $M D(n=37)$ & $0.020 \pm 0.01$ & $0.020 \pm 0.01$ & $0.021 \pm 0.01$ & $0.020 \pm 0.01$ & $\mathrm{Chi}^{2}=2.68 ; \mathrm{df}=3 ; p=0.44$ \\
\hline & $\operatorname{SFD}(n=25)$ & $0.023 \pm 0.01$ & $0.024 \pm 0.012$ & $0.023 \pm 0.01$ & $0.025 \pm 0.014$ & $\mathrm{Chi}^{2}=3.67 ; \mathrm{df}=3 ; p=0.30$ \\
\hline & $\mathrm{HC}(\mathrm{n}=43)$ & $0.021 \pm 0.01$ & $0.021 \pm 0.01$ & $0.021 \pm 0.01$ & $0.021 \pm 0.01$ & $\mathrm{Chi}^{2}=3.64 ; \mathrm{df}=3 ; p=0.30$ \\
\hline & Total $(n=105)$ & $0.021 \pm 0.01$ & $0.021 \pm 0.01$ & $0.022 \pm 0.01$ & $0.022 \pm 0.01$ & $\mathrm{Chi}^{2}=3.08 ; \mathrm{df}=3 ; p=0.38$ \\
\hline \multirow[t]{4}{*}{ Kynurenine/tryptophan } & $M D(n=37)$ & $27.03 \pm 8.45$ & $27.98 \pm 10.51$ & $27.45 \pm 8.08$ & $28.26 \pm 8.98$ & $\mathrm{Chi}^{2}=1.35 ; \mathrm{df}=3 ; p=0.72$ \\
\hline & $\operatorname{SFD}(n=26)$ & $30.20 \pm 13.36$ & $28.87 \pm 10.16$ & $30.72 \pm 13.50$ & $30.13 \pm 12.84$ & $\mathrm{Chi}^{2}=0.37 ; \mathrm{df}=3 ; p=0.95$ \\
\hline & $\mathrm{HC}(\mathrm{n}=42)$ & $29.44 \pm 10.49$ & $28.44 \pm 9.57$ & $28.91 \pm 9.66$ & $29.33 \pm 8.38$ & $\mathrm{Chi}^{2}=1.29 ; \mathrm{df}=3 ; p=0.73$ \\
\hline & Total $(n=105)$ & $28.78 \pm 10.62$ & $28.38 \pm 9.96$ & $28.85 \pm 10.24$ & $29.16 \pm 9.78$ & $\mathrm{Chi}^{2}=0.26 ; \mathrm{df}=3 ; p=0.97$ \\
\hline
\end{tabular}

Values are presented as mean \pm standard deviation.

$t 1$, month $1 ; t 2$, month $2 ; t 3$, month $3 ; t 4$, month 4 .

$\mathrm{MD}$, major depression; SFD, somatoform disorder; $\mathrm{HC}$, healthy control; df, degree of freedom. 
Table 3. Longitudinal analysis of cytokines

\begin{tabular}{clccccc}
\hline \multicolumn{2}{c}{ Biological parameter } & $t 1$ & $t 2$ & $t 3$ & $t 4$ & Friedman rank sum test \\
\hline IL-1 RA $(\mathrm{pg} / \mathrm{ml})$ & MD $(\mathrm{n}=37)$ & $359.9 \pm 320.9$ & $416.3 \pm 410.5$ & $324.9 \pm 224.1$ & $310.4 \pm 230.1$ & $\mathrm{Chi}^{2}=1.07 ; \mathrm{df}=3 ; p=0.78$ \\
& SFD $(\mathrm{n}=26)$ & $308.1 \pm 155.5$ & $330.1 \pm 203.3$ & $319.0 \pm 143.0$ & $316.9 \pm 150.2$ & $\mathrm{Chi}^{2}=0.26 ; \mathrm{df}=3 ; p=0.97$ \\
& HC $(\mathrm{n}=45)$ & $346.7 \pm 288.5$ & $343.3 \pm 204.0$ & $384.9 \pm 268.6$ & $391.99 \pm 299.2$ & $\mathrm{Chi}^{2}=4.7 ; \mathrm{df}=3 ; p=0.19$ \\
& Total $(\mathrm{n}=108)$ & $341.9 \pm 273.7$ & $365.1 \pm 291.3$ & $348.5 \pm 228.7$ & $373.6 \pm 295.4$ & $\mathrm{Chi}^{2}=0.77 ; \mathrm{df}=3 ; p=0.86$ \\
$\mathrm{IL}-6(\mathrm{pg} / \mathrm{ml})$ & MD $(\mathrm{n}=37)$ & $1.17 \pm 0.74$ & $1.78 \pm 2.57$ & $1.08 \pm 0.71$ & $1.12 \pm 0.83$ & $\mathrm{Chi}^{2}=4.87 ; \mathrm{df}=3 ; p=0.18$ \\
& SFD $(\mathrm{n}=26)$ & $1.55 \pm 2.12$ & $1.48 \pm 2.17$ & $1.63 \pm 2.29$ & $1.38 \pm 2.08$ & $\mathrm{Chi}^{2}=6.51 ; \mathrm{df}=3 ; p=0.09$ \\
& $\mathrm{HC}(\mathrm{n}=45)$ & $1.38 \pm 1.59$ & $1.47 \pm 1.85$ & $1.55 \pm 2.04$ & $1.62 \pm 2.00$ & $\mathrm{Chi}^{2}=0.66 ; \mathrm{df}=3 ; p=0.88$ \\
& Total $(\mathrm{n}=108)$ & $1.35 \pm 1.51$ & $1.58 \pm 2.18$ & $1.41 \pm 1.78$ & $1.39 \pm 1.72$ & $\mathrm{Chi}^{2}=2.59 ; \mathrm{df}=3 ; p=0.46$ \\
$\mathrm{TNF} \alpha(\mathrm{pg} / \mathrm{ml})$ & MD $(\mathrm{n}=37)$ & $1.41 \pm 0.44$ & $1.54 \pm 0.51$ & $1.48 \pm 0.48$ & $1.51 \pm 0.63$ & $\mathrm{Chi}^{2}=4.28 ; \mathrm{df}=3 ; p=0.23$ \\
& SFD $(\mathrm{n}=26)$ & $1.45 \pm 0.76$ & $1.45 \pm 0.76$ & $1.46 \pm 0.72$ & $1.43 \pm 0.65$ & $\mathrm{Chi}^{2}=0.70 ; \mathrm{df}=3 ; p=0.87$ \\
& HC $(\mathrm{n}=45)$ & $1.45 \pm 1.17$ & $1.36 \pm 0.54$ & $1.45 \pm 0.75$ & $1.39 \pm 0.63$ & $\mathrm{Chi}^{2}=1.26 ; \mathrm{df}=3 ; p=0.74$ \\
& Total $(\mathrm{n}=108)$ & $1.44 \pm 0.87$ & $1.44 \pm 0.59$ & $1.46 \pm 0.66$ & $1.44 \pm 0.34$ & $\mathrm{Chi}^{2}=1.92 ; \mathrm{df}=3 ; p=0.59$ \\
\hline
\end{tabular}

Values are presented as mean \pm standard deviation.

$t 1$, month $1 ; t 2$, month $2 ; t 3$, month $3 ; t 4$, month 4 .

IL, interleukin; RA, receptor antagonist; TNF, tumor necrosis factor; MD, major depression; SFD, somatoform disorder; HC, healthy control; df, degree of freedom.

Table 4. Correlation coefficients of cytokines and tryptophan metabolites at different time points

\begin{tabular}{|c|c|c|c|c|c|c|c|c|c|}
\hline & \multicolumn{3}{|c|}{ MD } & \multicolumn{3}{|c|}{ SFD } & \multicolumn{3}{|c|}{$\mathrm{HC}$} \\
\hline & $t 1-t 2$ & $t 1-t 3$ & $t 1-t 4$ & $t 1-t 2$ & $t 1-t 3$ & $t 1-t 4$ & $t 1-t 2$ & $t 1-t 3$ & $t 1-t 4$ \\
\hline Tryptophan & $0.41^{* *}$ & $0.65^{* *}$ & $0.57^{* *}$ & $0.70^{* *}$ & $0.54^{* *}$ & $0.42^{*}$ & $0.81^{* *}$ & $0.71^{* *}$ & $0.70^{* *}$ \\
\hline 5-HIAA & $0.81^{* *}$ & $0.88^{* *}$ & $0.71^{* *}$ & $0.80^{* *}$ & $0.96^{* *}$ & $0.73^{* *}$ & $0.90^{* *}$ & $0.90^{* *}$ & $0.87^{* *}$ \\
\hline Kynurenine & $0.71^{* *}$ & $0.64 * *$ & $0.64 * *$ & $0.86^{* *}$ & $0.86^{* *}$ & $0.88^{* *}$ & $0.74^{* *}$ & $0.61^{* *}$ & $0.55^{* *}$ \\
\hline 3-OH-kynurenine & $0.68^{* *}$ & $0.70^{* *}$ & $0.37^{*}$ & $0.67^{* *}$ & $0.79 * *$ & $0.82^{* *}$ & $0.69^{* *}$ & $0.71^{* *}$ & $0.53^{* *}$ \\
\hline Kynurenic acid & $0.87^{* *}$ & $0.77^{* *}$ & $0.81^{* *}$ & $0.64^{* *}$ & $0.82^{* *}$ & $0.81^{* *}$ & $0.62^{* *}$ & $0.76^{* *}$ & $0.70^{* *}$ \\
\hline IL-1-RA & $0.62^{* *}$ & $0.77^{* *}$ & $0.61^{* *}$ & $0.63^{* *}$ & $0.79^{* *}$ & $0.66^{* *}$ & $0.74^{* *}$ & $0.68^{* *}$ & $0.63^{* *}$ \\
\hline IL-6 & $0.48^{* *}$ & $0.68^{* *}$ & $0.67^{* *}$ & $0.65^{* *}$ & $0.61^{* *}$ & $0.73^{* *}$ & $0.53^{* *}$ & $0.53^{* *}$ & $0.66^{* *}$ \\
\hline TNF $\alpha$ & $0.85^{* *}$ & $0.86^{* *}$ & $0.82^{* *}$ & $0.95^{* *}$ & $0.88^{* *}$ & $0.87^{* *}$ & $0.76^{* *}$ & $0.74 * *$ & $0.62^{* *}$ \\
\hline
\end{tabular}

MD, major depression; SFD, somatoform disorder; HC, healthy controls; $t 1$, begin of examination; $t 2$, examination 4 weeks later; $t 3$, examination 8 weeks later; t4, examination 12 weeks later; 5-HIAA, 5-hydroxyindoleacetic acid; IL, interleukin; RA, receptor antagonist; TNF, tumor necrosis factor.

Spearman coefficient of correlation, $r ;{ }^{* *} p<0.01$.

Statistical analysis of BDI scores showed significant group differences between the patient groups and $\mathrm{HC}$ at all time points. A comparison of patient groups with the $t$ test revealed significant differences in depressive symptoms measured with the BDI at evaluations $t 1, t 2$, and $t 3$; differences at $t 4$ showed a trend towards significance. Statistical analysis of somatic symptoms (SOMS-7 sum) showed significant group differences between patient groups and $\mathrm{HC}$ at all evaluations. A comparison of SOMS-7 scores between patient groups revealed significant differences at $t 1$ and $t 3$; differences showed a trend towards significance at $t 2$, but at $t 4$ there was no such trend. SCL-90-R scores did not differ significantly between patient groups, although there was a trend towards higher scores in MD. Psychometric results are shown in detail in Table 5. Analysis of the psy- chometric scores with the Friedman rank sum test showed clear fluctuations over the 12 weeks in each of the groups. The only stable parameter was SOMS-7 in MD $(p=0.62)$ and $\mathrm{HC}(p=0.55)$.

\section{Relationship between the Kynurenine System and Cytokines}

There was a positive correlation between the kynurenine/TRP ratio and TNF $\alpha(\mathrm{r}=0.297, \mathrm{n}=84, p=0.007)$. For the other investigated cytokines IL-1 RA ( $r=0.177, \mathrm{n}=84$, $p=0.107)$ and IL-6 ( $\mathrm{r}=0.167, \mathrm{n}=84, p=0.129)$, no correlation to kynurenine/TRP ratio was found. Overall, increases in the KYN/TRP ratio were correlated with increases in TNF $\alpha$. 
Table 5. Psychometric results at each evaluation

\begin{tabular}{llcccc}
\hline \multicolumn{1}{c}{ Group } & $t 1$ & $t 2$ & $t 3$ & $t 4$ \\
\hline \multirow{2}{*}{ BDI } & MD $(\mathrm{n}=35-38)$ & $21.63 \pm 9.52$ & $18.45 \pm 9.23$ & $15.64 \pm 10.94$ & $15.24 \pm 9.73$ \\
& SFD $(\mathrm{n}=25-27)$ & $13.78 \pm 8.57$ & $11.62 \pm 7.56$ & $19.96 \pm 7.07$ & $10.77 \pm 8.05$ \\
& HC $(\mathrm{n}=45-51)$ & $3.06 \pm 4.25$ & $1.77 \pm 3.16$ & $1.41 \pm 2.93$ & $1.80 \pm 3.78$ \\
SCL-90R & MD vs. SFD & $\mathrm{T}=3.41 ; p=0.001^{*}$ & $\mathrm{~T}=3.12 ; p=0.003^{*}$ & $\mathrm{~T}=2.04 ; p=0.05^{*}$ & $\mathrm{~T}=1.93 ; p=0.06$ \\
& MD $(\mathrm{n}=35-38)$ & $90.57 \pm 45.06$ & $81.64 \pm 47.57$ & $67.23 \pm 46.85$ & $61.95 \pm 40.67$ \\
& SFD $(\mathrm{n}=25-27)$ & $70.13 \pm 47.13$ & $56.59 \pm 41.08$ & $51.04 \pm 35.61$ & $42.81 \pm 35.72$ \\
& HC $(\mathrm{n}=45-51)$ & $11.49 \pm 15.29$ & $9.69 \pm 12.33$ & $8.35 \pm 12.43$ & $8.80 \pm 12.95$ \\
SOMS-7 & MD vs. SFD & $\mathrm{T}=1.52 ; p=0.14$ & $\mathrm{~T}=1.92 ; p=0.06$ & $\mathrm{~T}=1.39 ; p=0.17$ & $\mathrm{~T}=1.94 ; p=0.06$ \\
& MD total $(\mathrm{n}=30-37)$ & $19.36 \pm 15.00$ & $13.05 \pm 16.48$ & $14.21 \pm 18.62$ & $18.59 \pm 18.91$ \\
& MD women $(\mathrm{n}=17-23)$ & $20.86 \pm 14.88$ & $22.55 \pm 17.97$ & $17.61 \pm 12.07$ & $18.61 \pm 12.37$ \\
& MD men $(\mathrm{n}=12-15)$ & $16.75 \pm 15.49$ & $19.62 \pm 19.50$ & $14.08 \pm 14.54$ & $18.57 \pm 27.04$ \\
& SFD total $(\mathrm{n}=19-27)$ & $29.31 \pm 17.45$ & $21.78 \pm 19.70$ & $25.59 \pm 19.30$ & $22.16 \pm 14.68$ \\
& SFD women $(\mathrm{n}=14-19)$ & $34.37 \pm 17.23$ & $29.83 \pm 18.31$ & $31.47 \pm 15.93$ & $24.32 \pm 15.54$ \\
& SFD men $(\mathrm{n}=5-7)$ & $15.57 \pm 8.77$ & $11.71 \pm 5.15$ & $17.00 \pm 9.95$ & $15.33 \pm 9.52$ \\
& HC total $(\mathrm{n}=43-45)$ & $5.86 \pm 5.63$ & $2.94 \pm 6.84$ & $2.78 \pm 7.20$ & $4.40 \pm 8.74$ \\
& HC women $(\mathrm{n}=29-31)$ & $6.09 \pm 5.65$ & $4.77 \pm 6.62$ & $4.90 \pm 6.42$ & $4.14 \pm 5.95$ \\
& HC men $(\mathrm{n}=13-16)$ & $5.38 \pm 5.75$ & $3.60 \pm 4.78$ & $3.29 \pm 3.25$ & $4.93 \pm 13.05$ \\
& MD vs. SFD & $\mathrm{T}=-2.4 ; p=0.02 *$ & $\mathrm{~T}=-2.0 ; p=0.055$ & $\mathrm{~T}=-2.4 ; p=0.02 *$ & $\mathrm{~T}=-0.79 ; p=0.43$ \\
\hline
\end{tabular}

Values are presented as mean \pm standard deviation.

Group differences were calculated with the $t$-test; * $p<0.05$ (statistically significant).

$t 1$, month 1 ; $t 2$, month $2 ; t 3$, month 3; $t 4$, month 4; BDI, Beck Depression Inventory; SCL-90-R, Symptom Checklist-90-Revised; SOMS-7, Screening for Somatoform Symptoms-7; MD, major depression; SFD, somatoform disorder; HC, healthy control.

\section{Correlations between Biological Parameters and Psychometric Test Results}

We performed a correlation analysis between the biological parameters and BDI, SCL-90-R, SOMS-7, and TICS-K. None of the investigated groups showed a stable association between a biological parameter and a psychometric variable at any of the assessment points.

\section{DISCUSSION}

The present study aimed to assess whether previous inconclusive psychobiological findings could be due to fluctuations of neurotransmitter and cytokine levels. In our study sample, we did not find significant fluctuations that might explain earlier incongruent psychobiological findings. The pro-inflammatory cytokines IL- 6 and TNF $\alpha$ and the anti-inflammatory cytokine IL-1 RA were stable in all three groups (MD, SFD, and $\mathrm{HC}$ ) over the 12-week study period, as were TRP, its kynurenine metabolites, and 5-HIAA. Concerning the stability of cytokines, a study in dialysis patients with chronic kidney disease, another illness associated with chronic inflammation, showed high fluctuations in IL-6 over a period of 12 weeks. ${ }^{27)}$ But a study on women with obesity showed a good within stability in a period of 3 years. ${ }^{28)}$ Previous studies have often only measured cytokines at one time point, whereas the psychopathology was assessed over years. Zalli et al. ${ }^{29}$ showed that IL- 6 and CRP levels at baseline could predict the persistence of depressive symptoms over 5 years.

We assume that differences might rather be related to characteristics of the study sample, like illness severity, illness duration, illness classification, co-morbidities, or sociodemographic parameters, i.e. BDI or physical activity level. ${ }^{30-33)}$ IL-1 RA has been found to be strongly elevated in people with obesity. ${ }^{34)}$ Accordingly the SFD group showed a significant correlation between BMI and both IL-6 and IL-1 RA. HC showed correlations between IL-6 and both $\mathrm{BMI}$ and age. This finding is concordant with that of an epidemiologic study on cytokines in the Swiss population, which showed that age and BMI correlate positively with IL- 6 and TNF $\alpha$. Further, the study showed higher TNF $\alpha$ in men who were smoking, but did not demonstrate any effect of physical activity in leisure time on cytokines like TNF $\alpha$ and IL-6. ${ }^{35)}$ In our study, we could also show an effect of smoking on TNF $\alpha$ in HC. Furthermore TNF $\alpha$ was the only cytokine that was positively correlated with KYN/TRP ratio that indicates the activity of the first degrading enzyme of TRP to kynurenine. This finding is in line with previous studies suggesting that increased TNF $\alpha$ correlates with increased monocyte, 
macrophage and microglial activation and therefore with the activity of the enzyme that breaks down TRP to kynurenine. ${ }^{36)}$

In addition to examining the biological parameters, we simultaneously measured the psychological state of health. We did not find any persistent correlations between biological parameters and depression (BDI), somatoform symptoms (SOMS-7), or global psychopathological impairment (SCL-90-R). But it has to be taken into account that immunological research is susceptible to artifacts and interfering variables.

This study has some limitations that might affect the generalizability of our results. First, the sample size of 117 (51 HC, 39 MD, and 27 SFD) was relatively small. Although the smallest group was that of patients with SFD, this group showed the highest stability of the investigated biological parameters. Second, patients in both patient groups were treated with antidepressants, which could have affected levels of neurotransmitters and cytokines. Moreover there are parameters in the kynurenine pathway, like quinolinic acid, which were not analysed in this study.

One strength of our study is the methodological approach; during the study period of 12 weeks, we specified an exact timeframe of four weeks between the measurements to consider possible effects of the menstrual cycle. Second, we excluded patients from the measurement if they had a common cold or any other medical condition. Third, we examined the interactions of cytokines and TRP-related neuroactive amines, instead of assessing only a single biological system. Fourth, we strictly separated $\mathrm{MD}$ and SFD, even though the two have a high comorbidity rate. Fifth, we recorded a large number of confounding factors.

Although many cross-sectional studies have focused on the "cytokine hypothesis" of depression as well as on its association with the monoamine hypothesis, in particular the kynurenine pathway, to our knowledge this study is the first to examine the longitudinal stability of TRP metabolites and cytokines in psychiatric patients in comparison to HC. Our data indicate that TRP metabolites and cytokines are reliable biological parameters in psychiatric research, because they do not fluctuate significantly in the course of time. This is also particular important because recent research found associations between depression and structural brain damage with increased risk for neuro- degenerative illnesses like Alzheimer disease. ${ }^{37)}$ This validates biological studies performed so far and will help to assess future studies in this field.

In our opinion, the major finding of our study is that fluctuations in cytokines such as IL-6, TNF $\alpha$, and IL-1 RA, and in TRP and its metabolites, 5-HIAA, 3-HK, and KYNA, are to a large extent negligible in MD and SFD.

\section{Acknowledgments}

This study was funded by a grant from the German Research Foundation (DFG). The DFG had no further role in the planning or execution of the study.

The authors thank Jacquie Klesing, ELS, for editing assistance with the manuscript. Further, the authors express their gratitude to all the participants for their great commitment to the study. The authors thank Johanna Zach for performing the analyses, Anina Burgbacher, Katharina Jacobi, and Benedikt Bedenk for data management, and Sebastian Meyer for statistical advice.

\section{REFERENCES}

1. Dantzer R, Bluthé RM, Castanon N, Kelley KW, Konsman JP, Laye $\mathrm{S}$, et al. Cytokines, sickness behavior, and depression. In: Robert A, editor. Psychoneuroimmunology. 4th ed. Burlington: Academic Press;2007. p.281-318.

2. Leonard BE. The concept of depression as a dysfunction of the immune system. Curr Immunol Rev 2010;6:205-212.

3. Dowlati Y, Herrmann N, Swardfager W, Liu H, Sham L, Reim EK, et al. A meta-analysis of cytokines in major depression. Biol Psychiatry 2010;67:446-457.

4. Howren MB, Lamkin DM, Suls J. Associations of depression with C-reactive protein, IL-1, and IL-6: a meta-analysis. Psychosom Med 2009;71:171-186.

5. Capuron L, Ravaud A, Neveu PJ, Miller AH, Maes M, Dantzer R. Association between decreased serum tryptophan concentrations and depressive symptoms in cancer patients undergoing cytokine therapy. Mol Psychiatry 2002;7:468-473.

6. Wichers MC, Koek GH, Robaeys G, Verkerk R, Scharpé S, Maes M. IDO and interferon-alpha-induced depressive symptoms: a shift in hypothesis from tryptophan depletion to neurotoxicity. Mol Psychiatry 2005;10:538-544.

7. Maes M, Leonard BE, Myint AM, Kubera M, Verkerk R. The new '5-HT' hypothesis of depression: cell-mediated immune activation induces indoleamine 2,3-dioxygenase, which leads to lower plasma tryptophan and an increased synthesis of detrimental tryptophan catabolites (TRYCATS), both of which contribute to the onset of depression. Prog Neuropsychopharmacol Biol Psychiatry 2011;35:702-721.

8. Demirci S, Aynal1 A, Demirci K, Demirci S, Arıdoğan BC. The serum levels of resistin and its relationship with other proin- 
flammatory cytokines in patients with alzheimer's disease. Clin Psychopharmacol Neurosci 2017;15:59-63.

9. Anderson G, Berk M, Maes M. Biological phenotypes underpin the physio-somatic symptoms of somatization, depression, and chronic fatigue syndrome. Acta Psychiatr Scand 2014;129:83-97.

10. Rief W, Hennings A, Riemer S, Euteneuer F. Psychobiological differences between depression and somatization. I Psychosom Res 2010;68:495-502.

11. Eyre H, Baune BT. Neuroimmunological effects of physical exercise in depression. Brain Behav Immun 2012;26:251266.

12. Kang HJ, Bae KY, Kim SW, Kim JT, Park MS, Cho KH, et al. Effects of interleukin-6, interleukin-18, and statin use, evaluated at acute stroke, on post-stroke depression during 1-year follow-up. Psychoneuroendocrinology 2016;72:156-160.

13. Tully PJ, Baumeister $H$, Bengel J, Jenkins A, Januszewski A, Martin S, et al. The longitudinal association between inflammation and incident depressive symptoms in men: the effects of hs-CRP are independent of abdominal obesity and metabolic disturbances. Physiol Behav 2015;139:328-335.

14. Euteneuer F, Schwarz MJ, Hennings A, Riemer S, Stapf T, Selberdinger $\mathrm{V}$, et al. Psychobiological aspects of somatization syndromes: contributions of inflammatory cytokines and neopterin. Psychiatry Res 2012;195:60-65.

15. Euteneuer F, Schwarz MJ, Hennings A, Riemer S, Stapf T, Selberdinger $\mathrm{V}$, et al. Depression, cytokines and experimental pain: evidence for sex-related association patterns. I Affect Disord 2011;131:143-9.

16. Wittchen HU, Zaudig M, Fydrich T. [Structured clinical interview for DSM-IVJ. Göttingen:Hogrefe Verlag;1997. German.

17. Hiller W, Rief W, Brähler E. Somatization in the population: from mild bodily misperceptions to disabling symptoms. SoC Psychiatry Psychiatr Epidemiol 2006;41:704-712.

18. Rief W, Henningsen P, Hiller W. Classification of somatoform disorders. Am J Psychiatry 2006;163:746-747; author reply 747-748.

19. Escobar JI, Rubio-Stipec M, Canino G, Karno M. Somatic symptom index (SSI): a new and abridged somatization construct. Prevalence and epidemiological correlates in two large community samples. J Nerv Ment Dis 1989;177:140146.

20. Rief W, Hiller W. Toward empirically based criteria for the classification of somatoform disorders. I Psychosom Res 1999; 46:507-518.

21. Schmitt M, Altstötter-Gleich C, Hinz A, Maes J, Brähler E. Norms for the simplified Beck Depression Inventory (BDI-V) in a non-clinical population. Diagnostica 2006;52:51-59.

22. Derogatis LR. SCL-90-R: Administration, scoring and procedures manual. 3rd ed. Minneapolis, MN:National Computer Systems; 1994.

23. Pedrosa Gil F, Nickel M, Ridout N, Schwarz MJ, Schoechlin C, Schmidmaier R. Alexithymia and interleukin variations in so- matoform disorder. Neuroimmunomodulation 2007;14:235242.

24. Frey I, Berg A, Grathwohl D, Keul J. [Freiburg Questionnaire of physical activity--development, evaluation and application]. Soz Praventivmed 1999;44:55-64. German.

25. Oades RD, Myint AM, Dauvermann MR, Schimmelmann BG, Schwarz MJ. Attention-deficit hyperactivity disorder (ADHD) and glial integrity: an exploration of associations of cytokines and kynurenine metabolites with symptoms and attention. Behav Brain Funct 2010;6:32.

26. Perneger TV. What's wrong with Bonferroni adjustments. BMJ 1998; 316:1236-1238.

27. LaClair R, O’ Neal K, Ofner S, Sosa MJ, Labarrere CA, Moe SM. Precision of biomarkers to define chronic inflammation in CKD. Am J Nephrol 2008;28:808-812.

28. Kaplan RC, Ho GY, Xue X, Rajpathak S, Cushman M, Rohan $\mathrm{TE}$, et al. Within-individual stability of obesity-related biomarkers among women. Cancer Epidemiol Biomarkers Prev 2007; 16:1291-1293.

29. Zalli A, Jovanova O, Hoogendijk WJ, Tiemeier H, Carvalho LA. Low-grade inflammation predicts persistence of depressive symptoms. Psychopharmacology (Berl) 2016;233:16691678.

30. Maes M, Berk M, Goehler L, Song C, Anderson G, Gałecki P, et al. Depression and sickness behavior are Janus-faced responses to shared inflammatory pathways. BMC Med 2012; 10:66.

31. Dantzer R, O'Connor JC, Lawson MA, Kelley KW. Inflammationassociated depression: from serotonin to kynurenine. Psychoneuroendocrinology 2011;36:426-436.

32. Gabbay V, Liebes L, Katz Y, Liu S, Mendoza S, Babb JS, et al. The kynurenine pathway in adolescent depression: preliminary findings from a proton MR spectroscopy study. Prog Neuropsychopharmacol Biol Psychiatry 2010;34:37-44.

33. Rothermundt $M$, Arolt V, Fenker J, Gutbrodt $H$, Peters $M$, Kirchner H. Different immune patterns in melancholic and non-melancholic major depression. Eur Arch Psychiatry Clin Neurosci 2001;251:90-97.

34. Meier CA, Bobbioni E, Gabay C, Assimacopoulos-Jeannet F, Golay A, Dayer JM. IL-1 receptor antagonist serum levels are increased in human obesity: a possible link to the resistance to leptin? J Clin Endocrinol Metab 2002;87:1184-1188.

35. Marques-Vidal $P$, Bochud $M$, Bastardot $F$, Lüscher T, Ferrero $F$, Gaspoz JM, et al. Levels and determinants of inflammatory biomarkers in a Swiss population-based sample (CoLaus study). PLoS One 2011;6:e21002.

36. Kwidzinski E, Bechmann I. IDO expression in the brain: a double-edged sword. J Mol Med (Berl) 2007;85:1351-1359.

37. Moylan S, Maes M, Wray NR, Berk M. The neuroprogressive nature of major depressive disorder: pathways to disease evolution and resistance, and therapeutic implications. Mol Psychiatry 2013;18:595-606. 